\title{
Bedeutung des Immunsystems bei Infektionen und die Beeinflussung durch Pflanzen
}

\author{
Sabine Vollstedt
}

Das Haustier ist dem Menschen heutzutage näher als je zuvor und sollte bei jeglicher gesundheitlicher Evaluation mit bedacht werden. Die Rolle der Zoonosen wie auch der Übertragung resistenter Bakterienstämme stellt uns vor eine Realität, mit der wir noch lernen müssen, wie wir am besten damit umgehen. Prävention allein reicht nicht mehr aus, sondern wir müssen den Schritt vorher den zum präventiven Denken - vollziehen, um mit den Gegebenheiten fertig zu werden. Das Wissen, welche Strukturen auf welche Weise gestärkt werden können, ist immens wichtig, sowohl beim Menschen wie auch beim Tier.

\section{Das Immunsystem}

Das Immunsystem gehört mit zu den wichtigen Strukturen im Körper, weil es dafür sorgt, dass Pathogene nicht eindringen und somit Schaden anrichten können. Es gehören viele Strukturen dazu, die oftmals nicht bedacht werden. So sind Körperoberflächen wie die Haut und die Schleimhäute Teil des Immunsystems, wie das Interstitium mit den Lymphbahnen, das Gefäßsystem mit den Kapillaren und natürlich auch die Lymphknoten ( Abb. 1).

\section{Angeborene und erworbene Abwehrsysteme}

Die Immunzellen sind die zweite Komponente, die dafür sorgen, dass die Abwehr effektiv erfolgen kann. Hier wird zwischen dem angeborenen Immunsystem mit den Dendritischen Zellen (DC), Makrophagen und Natürlichen Killerzellen (NK) und dem erworbenen Immunsystem wie B-Zellen, Plasmazellen und T-Zellen unterschieden.

Die Zellen des unspezifischen Immunsystems befinden sich oft in der Peripherie, dort wo eine Infektion droht, während die naiven B- und T-Zellen sich in den Lymphknoten befinden und erst zum Ort des Infektionsgeschehens auswandern, wenn sie einen entsprechenden Rezeptor besitzen, der zum aktuellen Pathogen passt [1].

\section{Humorale Immunität}

Da es eine Vielzahl von Pathogenen gibt, sind die Immunantworten entsprechend divers. Extrazelluläre Pathogene können Endoparasiten, Bakterien, Pilze, Protozoen und auch Viren sein. B-Zellen spielen eine große Rolle, indem sie sich zu Plasmazellen wandeln, um große Mengen an Antikörper zu produzieren, die genau auf das entsprechende Pathogen wirken. Aber auch Makrophagen sind wichtig, da diese die mit Antikörpern versehenen Pathogene durch Phagozytose eliminieren. Dieses Zusammenspiel wird als humorale Immunität beschrieben oder auch als TH2-Antwort, weil die T-Helferzellen Typ 2 bei der Produktion von Antikörpern eine wichtige, unterstützende Rolle spielen [1].

\section{Zelluläre Immunität}

Bei den intrazellulären Pathogenen wird hauptsächlich zwischen Viren und intrazellulären Bakterien unterschieden. Zur Abwehr von Viren sind sowohl Natürliche Killerzellen (NK) wie auch zytotoxische T-Zellen wichtig, weil sie die befallenen Körperzellen abtöten, sodass diese nicht mehr als Produktionsstätte für die Viren genutzt werden können. Bei den intrazellulären Bakterien kommen hauptsächlich NK-Zellen und T-Helferzellen Typ 1 zum Zuge. NK-Zellen lysieren die befallenen Zellen und die TH1-Zellen produzieren Botenstoffe, sogenannte Zytokine, die für eine Verstärkung der Makrophagenaktivität sorgen, sodass diese ihre Phagozytose noch effizienter durchführen können [1].

In den letzten Jahren sind außerdem noch zwei weitere T-Zellen in den Fokus der Wissenschaft geraten: Die eine ist die T-Zelle Typ 17, die Interleukin-17 produziert und dadurch in der Mukosa Entzündungsreaktionen hervorruft. Die zweite ist die regulierende T-Zelle (Treg), die aktivierte T-Zellen wieder inhibieren kann und somit Entzündungsreaktionen beendet [2].

\section{Dendritische Zellen als Dirigenten}

Die Entwicklung dieser unterschiedlichen TH-Zellen hängt von den Zytokinmustern ab, die - insbesondere 


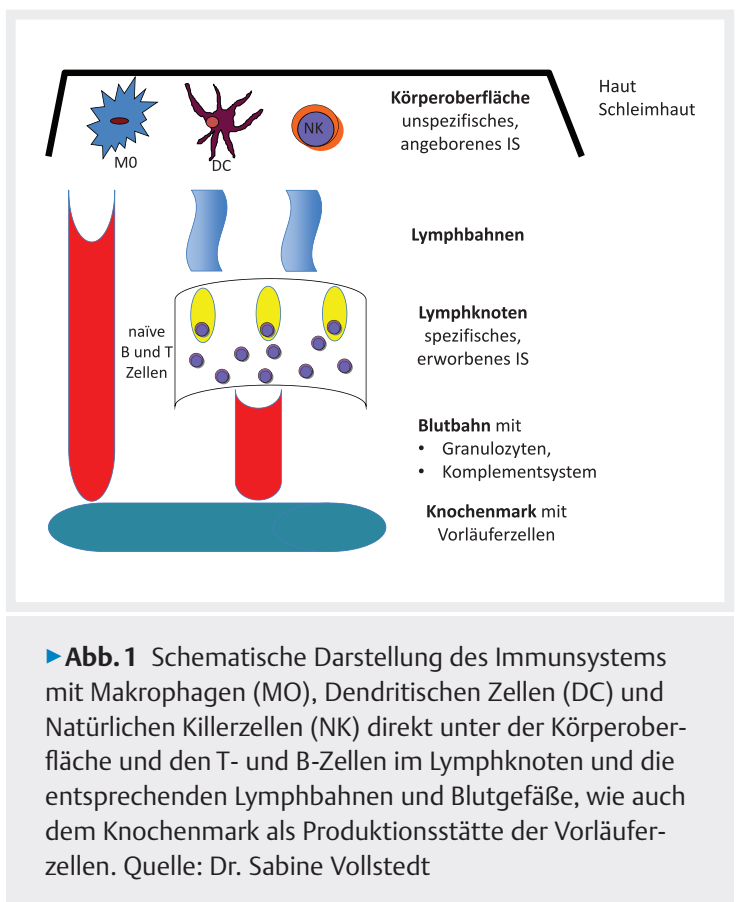

von den Dendritischen Zellen (DC) - am Anfang einer Infektion produziert werden.

DC sind Wächterzellen, die dort sitzen, wo Infektionen stattfinden, d.h. besonders im Bereich der Schleimhäute des Magen-Darm-Traktes, des Respirationstraktes oder auch in der Haut. Sie sind spezialisiert und mit Rezeptoren ausgestattet, die es ermöglichen, unterschiedliche pathogene Oberflächenstrukturen zu erkennen. Im Fall einer intrazellulären Infektion mit Bakterien oder Viren wird lokal viel TNF- $\alpha$ ausgeschüttet. Wenn es Bakterien sind, kommt noch IL-12 dazu, bei Viren IFN- $\alpha, \beta$. Bei Vorhandensein extrazellulärer Bakterien wird IL-4 produziert. Diese Zytokinproduktion bestimmt dementsprechend auch die Entwicklung der Immunantwort. Um eine TH1Antwort zu induzieren, braucht es hohe Mengen an IL-12, für eine TH2-Antwort ist es das IL-4. Wenn die DC viel IL23 ausschütten, entsteht hauptsächlich eine TH17-Antwort und bei hohen Mengen an TGF- $\beta$ kommt es zu einer Aktivierung der regulierenden T-Zellen. Somit sind die DC in der Lage, die Immunantwort entsprechend zu modulieren und dem Infektionsgeschehen anzupassen [3].

\section{Immunmodulation}

Das mukosale Immunsystem besitzt einige Strukturen, die für eine ausgewogene Immunantwort wichtig sind. Die Verbindung des mukosalen Epithels mit den Immunzellen ist sehr eng, und daraus entstehen besondere Strukturen wie die Peyer'schen Platten oder auch die MZellen als spezialisiertes Aufnahmesystem von Bakterien im Darm ( $\mathbf{A}$ bb.2). Im Magen-Darm-Trakt gibt es aktivierte Gedächtniszellen und aktivierte unspezifische

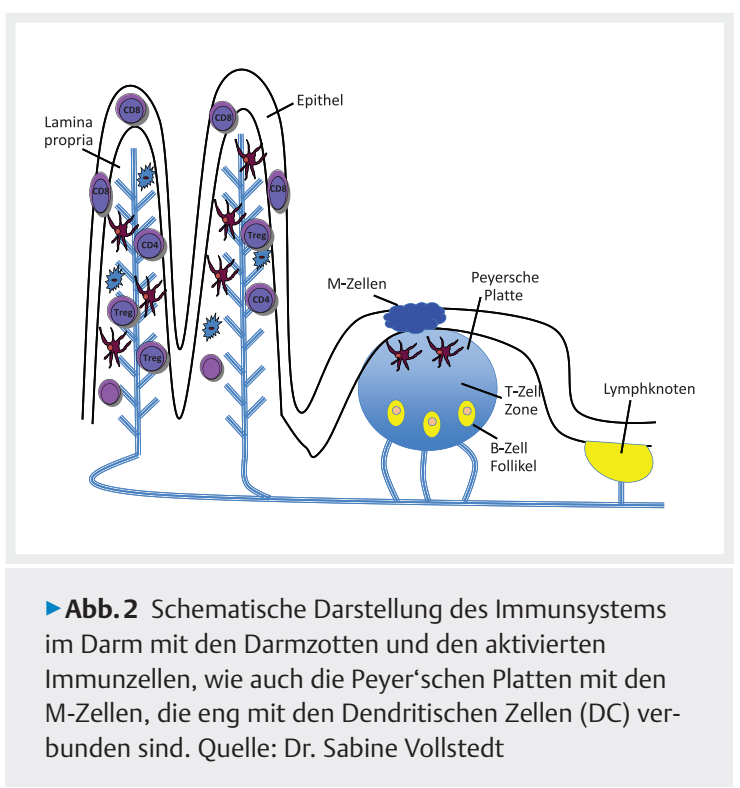

Zellen, auch wenn es noch keinen Anlass für eine sofortige Immunreaktion gibt. Sollten pathogene Erreger die Überhand nehmen, können sie eine sofortige Immunreaktion initiieren. Daher hat die aktive Inhibierung dieser aktiven Zellen eine große Bedeutung, denn sonst würden Futterbestandteile und das Mikrobiom ständig eine Immunantwort auslösen. Daher finden sich inhibierende Makrophagen wie auch toleranz-induzierende DC in hohen Mengen im Magen-Darm-Trakt [2].

\section{Mikrobiom}

Es ist wichtig zu wissen, dass jeder Keim, egal ob pathogen oder nicht, eine Immunantwort auslöst. Im Darmepithel gibt es viele aktivierte zytotoxische T-Zellen, die ständig durch regulierende T-Zellen kontrolliert werden müssen, da es ansonsten zu massiven Schäden der Darmschleimhaut käme. Die DC mit ihren sehr gut definierten Zytokinmustern orchestrieren dieses feine Zusammenspiel der unterschiedlichen Immunzellen. Dadurch können die vielzähligen Bakterien des Mikrobioms ihre wichtigen Aufgaben wie Aufschluss von Futter, Verdauung von Nahrungsbestandteilen und Anregung der Darmperistaltik wahrnehmen, sodass das Darmepithel mit Energie und auch Vitaminen versorgt wird. Das Mikrobiom dient somit als Trainingsgrundlage für das Immunsystem und sorgt dafür, dass sich pathogene Keime nicht ausbreiten können, weil Bakterien und Pilze des Mikrobioms den vorhandenen Platz schon belegen [2].

\section{Interaktion zwischen}

\section{Phytotherapeutika und Immunsystem}

In der Phytotherapie sind wir in der Lage, uns diese Grundlage zunutze zu machen, weil der größte Teil der Phytotherapeutika oral aufgenommen wird und dann 
den MDT durchläuft. Die sekundären Pflanzenstoffe wirken hierbei nicht nur inhibierend auf die pathogenen Keime, sondern sind auch in der Lage, spezifische Rezeptoren der Immunzellen anzusprechen, um dann entsprechende Zytokinmuster hervorzurufen ( $>$ Abb. 3).

Diese Zytokinmuster beziehen sich auf die Infektionslage. Bei einer zellulären Immunantwort werden pro-entzündliche Zytokine wie IL-6, IL-8 und IL-12 produziert, während bei einer humoralen Immunantwort IL-4, IL-10 und TGF- $\beta$ ausgeschüttet werden. Die Produktion eines bestimmten Zytokins ist abhängig von der Aktivierung definierter Rezeptoren. Interagieren Rezeptoren direkt mit den Keimen (pathogen-associated molecular pattern - PAMP) und auch Toll-like receptor-2 und -4 (TLR-2, TLR-4), werden pro-entzündliche Zytokinmuster hervorgerufen. Der Adenosin-Rezeptor dagegen aktiviert eine anti-entzündliche Kaskade von Zytokinen [4].

\section{Sekundäre Pflanzenstoffe}

Flavonoide sind in der Lage, sowohl mit TLR-2, dem TLR4 wie auch mit dem Adenosin- und dem Aryl-Hydrocarbon(Ah)-Rezeptor zu interagieren. Diese Rezeptoren bewirken sowohl pro- wie auch anti-entzündliche Zytokinmuster und scheinen damit die Entzündungsprozesse zu modulieren [5]. Mädesüß ( $\triangleright$ Abb.4), Stiefmütterchen und Mariendistel ( $\triangleright$ Abb.5) sind nur einige Beispiele, die dafür bekannt sind, überschießende Entzündungsreaktionen herabregulieren zu können.

Polysaccharide sind bekannt dafür, dass sie in Verbindung mit Flüssigkeit Schleim bilden und sich somit schützend über geschädigte Schleimhautläsionen legen können. Sie wirken ebenso pro-entzündlich, da sie aktiv

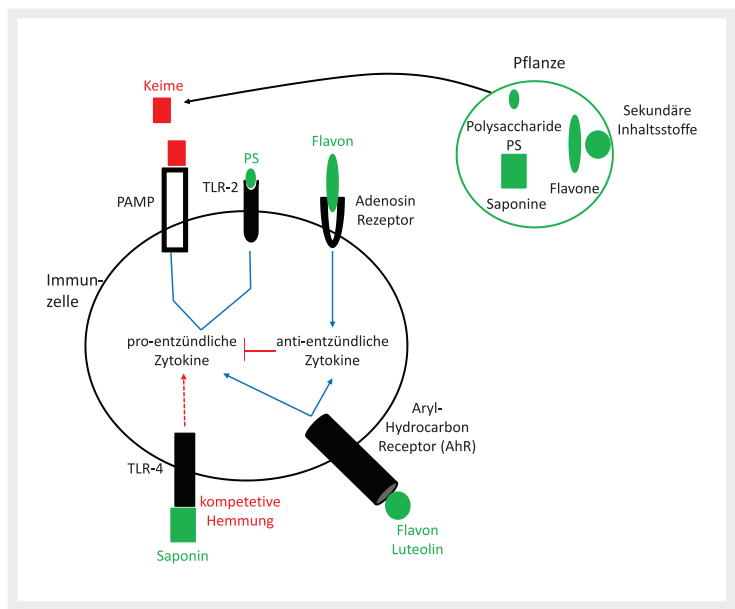

- Abb. 3 Schematische Darstellung der Interaktionen von sekundären Inhaltsstoffen mit Rezeptoren auf Immunzellen mit einer anschließenden Induktion von pro- oder anti-entzündlichen Zytokinmustern. Quelle: Dr. Sabine Vollstedt

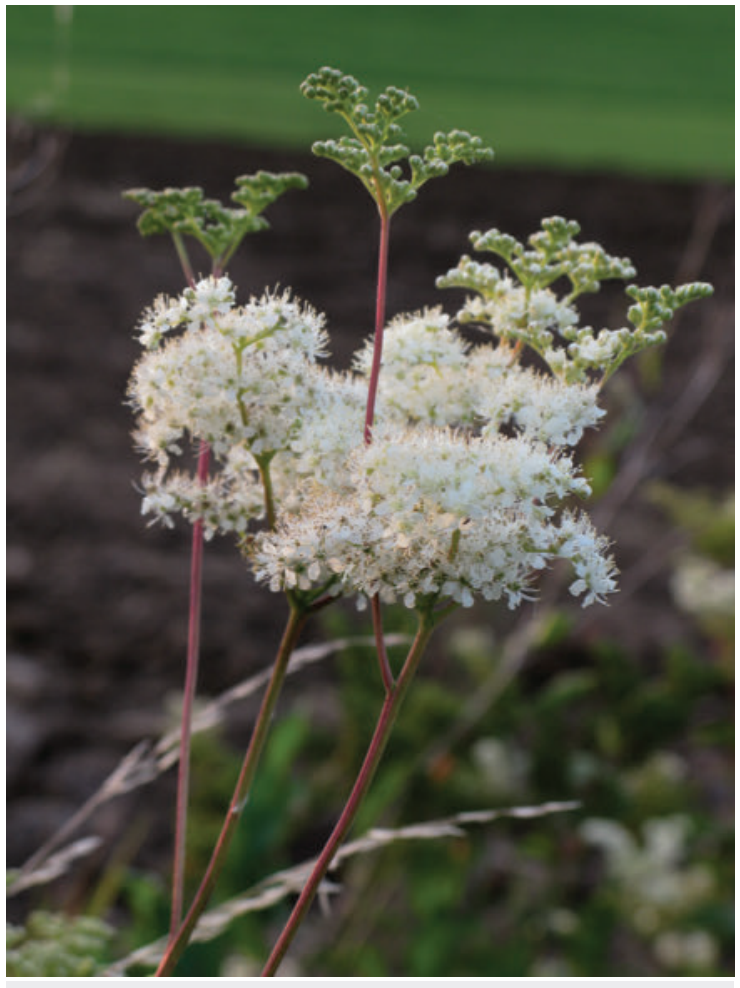

-Abb. 4 Mädesüß (Filipendula ulmaria). Quelle: Dr. Ferdinand Worm

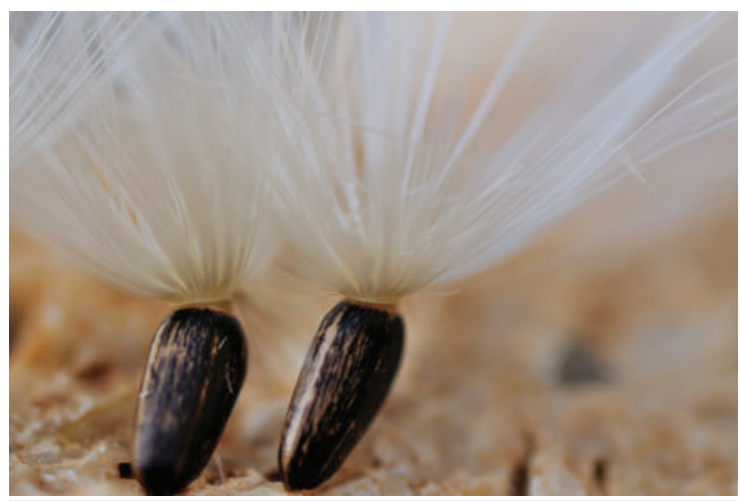

-Abb.5 Mariendistel (Silybum marianum): Früchte mit charakteristischem Pappus. Quelle: Dr. Ferdinand Worm

den TLR-4 aktivieren und damit entsprechende Zytokine freigesetzt werden [6]. Beispiele für Polysaccharidpflanzen sind Leinsamen, Bockshornkleesamen ( $\mathbf{A} \mathbf{A b} \mathbf{b} \mathbf{6}$ ), Isländisch Moos und Malve.

Saponine können Oberflächenspannungen lösen und werden dementsprechend therapeutisch eingesetzt. Es gibt noch weitere Funktionen, denn einerseits setzen sie sich an den TLR-4 und blockieren ihn so für andere Agonisten mit dem Ergebnis, dass keine pro-entzündlichen Zytokine produziert werden können. Andererseits wirken Saponine auch sedierend im ZNS, wahrscheinlich durch 

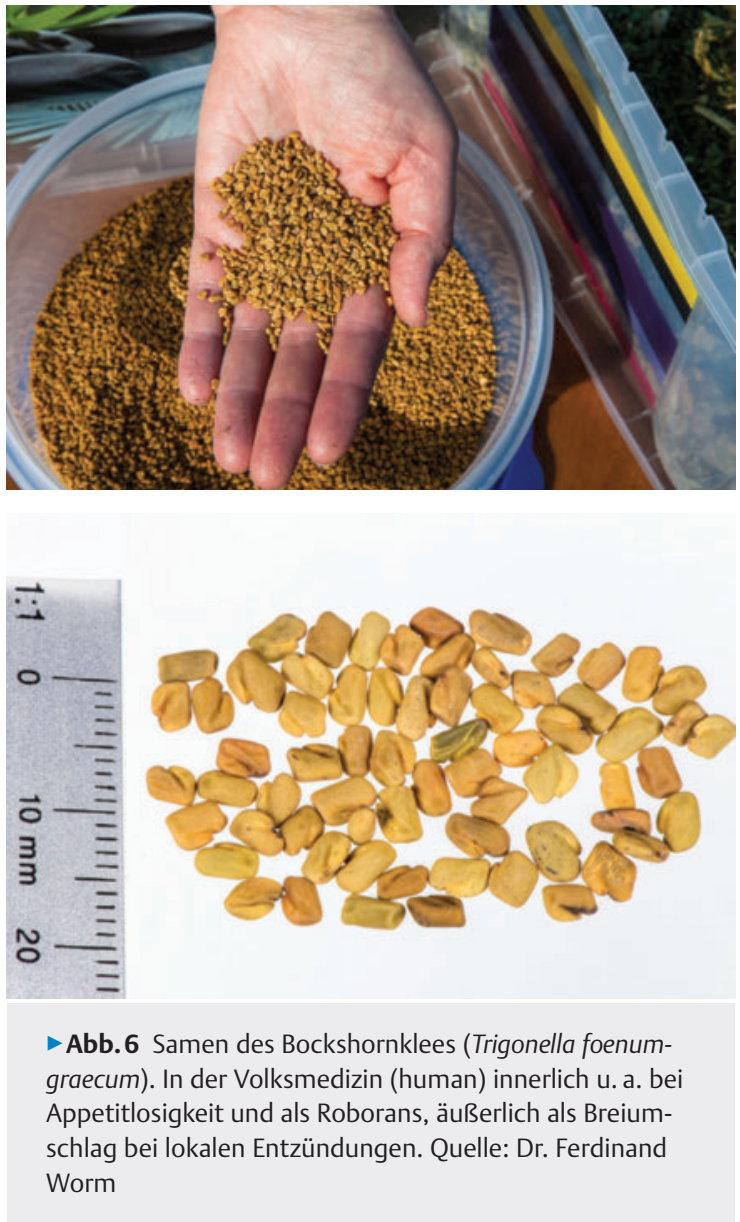

eine Interaktion mit dem 5- $\mathrm{HT}_{2}$-Rezeptor. Pflanzen mit hohem Saponingehalt sind Ginseng, Efeublätter, Ringelblume und Süßholzwurzel [7], [8], [9].

\section{Phytotherapie}

Phytotherapeutisch ist es möglich, positiv auf die Darmgesundheit einzuwirken, bevor Symptome auftreten. Die vielseitige und ausgeglichene Aktivierung der unterschiedlichen Rezeptoren auf den Immunzellen wirkt modulierend und ausgleichend und ist daher auch für die Prävention gut einsetzbar. Bei einer Erkrankung, wie etwa einer Endoparasitose, unterstützen die Pflanzen die Immunzellen dahingehend, dass sie eine effektive Immunantwort hervorrufen. Davon abgesehen wirken einige Pflanzen auch direkt auf die Pathogene, wie Endoparasiten. Die Kombination von Schulmedizin mit der Phytotherapie ist daher sehr sinnvoll. Ebenso dienen die Pflanzen direkt nach einer Behandlung der Rekonvaleszenz, nicht auch zuletzt, weil sie den Nebenwirkungen einiger unserer synthetischen Arzneimittel entgegenwirken.

\section{Fazit}

Phytotherapeutika sind daher vielseitig und zu jedem Zeitpunkt einsetzbar und ermöglichen eine genaue und feinere Behandlungsstrategie, um die Gesunderhaltung der Tiere zu unterstützen.

Interessenkonflikt

Die Autorin erklärt, dass kein Interessenkonflikt besteht.

Korrespondenzadresse

Dr. med. vet. Ph.D. Sabine Vollstedt

Hauptstr. 53

25335 Bokholt-Hanredder

Deutschland

E-Mail: tcpm@svollstedt.com

\section{Literatur}

[1] Murphy K et al. Janeway's Immunobiology. 7th ed. New York and London: Garland Science; 2008

[2] Belkaid Y, Harrison OJ. Homeostatic immunity and the microbiota. Immunity 2017; 46: 562-576. doi:10.1016/j. immuni.2017.04.008

[3] Devi KS, Anandasabapathy N. The origin of DCs and capacity for immunologic tolerance in central and peripheral tissues. Semin Immunopathol 2017; 39: 137-152. doi:10.1007/ s00281-016-0602-0

[4] Lim KH, Staudt LM. Toll-like receptor signaling. Cold Spring Harb Perspect Biol 2013; 5: a011247. doi:10.1101/cshperspect.a011247

[5] Kim J, Durai P, Jeon D et al. Phloretin as a potent natural TLR2/1 inhibitor suppresses TLR2-induced inflammation. Nutrients 2018; 10: 868. doi:10.3390/nu10070868

[6] Jiao R, Liu Y, Gao $\mathrm{H}$ et al. The anti-oxidant and antitumor properties of plant polysaccharides. Am J Chin Med 2016; 44: 463-488

[7] Silva A, Mount A, Krstevska K et al. The combination of ISCOMATRIX adjuvant and TLR agonists induces regression of established solid tumors in vivo. I Immunol 2015; 194: 21992207. doi:10.4049/jimmunol.1402228

[8] Sun CL, Geng CA, Huang XY et al. Bioassay-guided isolation of saikosaponins with agonistic activity on 5-hydroxytryptamine $2 \mathrm{C}$ receptor from Bupleurum chinense and their potential use for the treatment of obesity. Chin J Nat Med 2017; 15: 467473. doi:10.1016/S1875-5364(17)30070-5

[9] Schmidt S, Heimesaat MM, Fischer A et al. Saponins increase susceptibility of vancomycin-resistant enterococci to antibiotic compounds. Eur J Microbiol Immunol (Bp)2014; 4: 204212. doi:10.1556/EUJMI-D-14-00029

Bibliografie

Zeitschrift für Phytotherapie 2021; 42: 5-8

DOI 10.1055/a-1338-2314

ISSN 0722-348X

(c) 2021. Thieme. All rights reserved.

Georg Thieme Verlag KG, Rüdigerstraße 14,

70469 Stuttgart, Germany 